\title{
A biossegurança em tempos de covid-19 no Curso de Odontologia da Unimontes: relato de experiência
}

\section{Biosafety in times of covid-19 at the Dental School of Unimontes: experience report}

\author{
Gabriel Felipe Albuquerque Barbosa ${ }^{1}$ \\ Amanda Fróes Ribeiro ${ }^{2}$ \\ Maria Cleonice de Oliveira Nobre ${ }^{3}$ \\ Renata Francine Rodrigues Lima 4 \\ Carolina de Castro Oliveira ${ }^{5}$ \\ Maria de Lourdes Carvalho Bonfim ${ }^{6}$ \\ Soraya Mameluque Ferreira ${ }^{7}$ \\ Mânia de Quadros Coelho Pinto ${ }^{8}$
}

\footnotetext{
${ }^{1}$ Acadêmico de Odontologia da Universidade Estadual de Montes Claros (Unimontes). Montes Claros - MG. Brasil. $₫$ gabriel-barbosa12@hotmail.com (i) https://orcid.org/0000-0003-3351-7973.

${ }^{2}$ Acadêmica de Odontologia da Universidade Estadual de Montes Claros (Unimontes). Montes Claros - MG. Brasil. $\varangle$ froesribeiro10@gmail.com (i) https://orcid.org/0000-0002-3523-5016.

${ }^{3}$ Especialista em Administração dos Serviços de Saúde (UNAERP). Professora da Universidade Estadual de Montes Claros (Unimontes). Montes Claros - MG. Brasil. $\square$ cleonicenobre@ yahoo.com.br

(i) https://orcid.org/0000-0001-9139-1278.

${ }^{4}$ Doutora em Ciências da Saúde (Unimontes). Professora da Universidade Estadual de Montes Claros (Unimontes). Montes Claros - MG. Brasil. $₫$ renata.oliveira@unimontes.br (D) https://orcid.org/0000-00027393-0415.

${ }^{5}$ Doutora em Odontologia (UNICSUL). Professora da Universidade Estadual de Montes Claros (Unimontes). Montes Claros - MG. Brasil. $₫$ carolina.oliveira@unimontes.br (i) https://orcid.org/0000-0002-9620-9989

${ }^{6}$ Doutora em Odontologia em Saúde Coletiva (UFMG). Professora da Universidade Estadual de Montes Claros (Unimontes). Montes Claros - MG. Brasil. $₫$ maria.bonfim@unimontes.br $@$ https://orcid.org/0000-00016399-3212.

${ }^{7}$ Doutora em Clínica Restauradora (FORP-USP). Professora da Universidade Estadual de Montes Claros (Unimontes). Montes Claros - MG. Brasil. $\triangle$ soraya@mameluque.com.br (i) https://orcid.org/0000-00015711-9473.

${ }^{8}$ Doutora em Ciências da Saúde (Unimontes). Professora da Universidade Estadual de Montes Claros (Unimontes). Montes Claros - MG. Brasil. $\triangle$ mania.coelho@unimontes.br (i) https://orcid.org/0000-00026913-8695.
}

$\begin{array}{ccc}\text { Recebido em } & \text { Aceito em } & \text { Publicado em } \\ 11 / 10 / 2020 & 03 / 12 / 2020 & 31 / 12 / 2020\end{array}$


Resumo: a pandemia pela nova cepa de coronavírus, o SARS-CoV-2, modificou a forma de atendimento de muitas profissões e serviu de alerta para a necessidade de constante atualização dos profissionais e normas de biossegurança. Objetivo: relatar a experiência da Comissão de Biossegurança e Infraestrutura do Curso de Odontologia da Universidade Estadual de Montes Claros - Unimontes na atuação e criação de um novo protocolo de biossegurança, durante a pandemia da COVID-19. Relato de Experiência: Embora existisse um manual previamente estabelecido e normatizado, tornou-se necessário o desenvolvimento de um novo documento. Foi realizada uma análise criteriosa da literatura, leis, portarias e normas técnicas do Ministério da Saúde, Secretarias Estaduais e Municipais, Protocolos de Biossegurança de outras Instituições de Ensino Superior (IES) e de Entidades de Classe. Após diversas reuniões online, de março a julho de 2020, elaborou-se o Protocolo de Biossegurança do Curso de Odontologia da Unimontes em Tempos de COVID-19. Conclusão: Os cuidados à saúde são de extrema importância para reduzir o risco de infecção cruzada, sobretudo no momento de pandemia. Espera-se que esse documento seja utilizado na adequação do cuidado com a segurança necessária, tendo em vista a retomada das atividades presenciais do curso de Odontologia.

Palavras-chave: Contenção de Riscos Biológicos; Odontologia; COVID-19.

Abstract: The pandemic due to the new coronavirus strain, SARS-CoV-2, changed the way of working of many professions and served as a warning to the need for constant updating of professionals and biosafety standards. Objective: Report the experience of the Biosafety and Infrastructure Commission of the Dentistry Course at the State University of Montes Claros - Unimontes, in the performance and creation of a new biosafety protocol during the COVID-19 pandemic. Experience Report: Although there was a previously established and standardized guide, it was necessary to develop a new document. A thorough analysis of the literature, laws, ordinances and technical standards of the Ministry of Health, State and Municipal Secretariats, Biosafety Protocols of other Higher Education Institutions and Class Entities was carried out. After several online meetings, from March to July 2020, the Biosafety Protocol for the Dentistry Course at Unimontes in Times of COVID-19 was developed. Conclusion: Health care is extremely important to reduce the risk of crossinfection, especially in a time of pandemic. It is expected that this document will be used in the adequacy of care with the necessary safety, in view of the resumption of presential activities in the Dentistry course.

Keywords: Containment of Biohazards; Dentistry; COVID-19.

\section{INTRODUÇÃO}

Os trabalhadores, de maneira geral, estão constantemente expostos aos diferentes riscos ocupacionais, como àqueles causados por acidentes, ergonômicos e por agentes químicos, físicos e biológicos. No caso dos profissionais da saúde, esse último é de 
importância primordial em seu cotidiano, visto que estão presentes rotineiramente em seus ambientes de trabalho ${ }^{1}$.

Entretanto, foi a partir da década de 1980 o início das primeiras discussões e estudos acerca da biossegurança relacionada à Odontologia, quando veio à tona a epidemia da Síndrome da Imunodeficiência Adquirida (SIDA), quando houve o primeiro relato de contaminação por acidente de trabalho em saúde ${ }^{2}$. A necessidade de controle de infecção em Odontologia alertou a comunidade de saúde sobre o perigo de transmissão ocupacional de agentes infecciosos, obrigando esse setor a aperfeiçoar os métodos de prevenção de risco de contaminação adotados pelos cirurgiões-dentistas, o que impulsionou maior preocupação com a biossegurança ${ }^{3}$.

Além disso, com a globalização e o desenvolvimento tecnológico, o fluxo de pessoas por diferentes cidades, estados e países rompeu fronteiras antes existentes e contribuiu para que microrganismos patógenos não se restringissem apenas a determinados locais; fenômeno este que ascendeu, ainda mais, a preocupação com a disseminação de doenças e infecções, levando pesquisadores e autoridades a desenvolver políticas públicas e estratégias em áreas específicas, com o intuito de se minimizar os danos à saúde da população ${ }^{1,4}$.

$\mathrm{Na}$ atualidade, a exemplo dessa constante movimentação populacional, o mundo enfrenta, desde o final de 2019, a pandemia pela nova cepa de coronavírus, o SARS-CoV-2, cuja doença denomina-se COVID-19. Constituindo uma emergência de saúde pública global, a pandemia modificou a forma de atendimento de muitas profissões e serviu de alerta para a necessidade de constante atualização dos profissionais no sentido de se conhecer formas de controle de infecção, assim como, quais os produtos disponíveis e procedimentos a serem adotados para essa finalidade ${ }^{5}$.

O contágio pode ocorrer por transmissão direta (fluidos corporais) ou indireta (pelo contato com superfícies contaminadas) ${ }^{6}$. O fato de o SARS-CoV-2 ser identificado na saliva de pacientes infectados é um lembrete de que os cirurgiões-dentistas devem atentarse à disseminação de doenças infecciosas respiratórias, sobretudo na formação de aerossóis, durante o atendimento odontológico 5 .

Nesse sentido, a Comissão de Biossegurança e Infraestrutura do Curso de Odontologia da Universidade Estadual de Montes Claros - Unimontes mobilizou-se com o propósito de compreender as novas demandas estruturais, de materiais, equipamentos e 
condutas a fim de elaborar um documento para nortear as atividades acadêmicas nos Laboratórios e Clínicas Odontológicas dessa Universidade.

Portanto, o objetivo deste estudo é relatar a experiência dessa Comissão frente à nova realidade instaurada pela pandemia da COVID-19, assim como na criação de um novo protocolo de biossegurança a ser adotado na prática clínica.

\section{RELATO DE EXPERIÊNCIA}

Diante das recomendações da Associação Brasileira de Ensino Odontológico ABENO, Ministério da Educação - MEC, Agência Nacional de Vigilância Sanitária - Anvisa e Ministério da Saúde, o Curso de Odontologia da Unimontes instituiu uma Comissão de Controle de Infecção/Biossegurança, para organização de uma prática clínica odontológica mais adequada aos moldes exigidos nos tempos atuais.

Assim, em 14 de setembro de 2010, foi publicada a Portaria no 094 - Reitor/2010 que formalizou a Comissão de Biossegurança e Infraestrutura das Clínicas e Laboratórios do Curso de Odontologia ${ }^{7}$. Atualmente, é constituída por 10 membros, sendo 07 do corpo docente, 01 servidora pública e 02 acadêmicos do curso.

A Comissão, embasada nas resoluções e recomendações dos órgãos supracitados, elaborou um manual de esclarecimentos e orientações sobre biossegurança no ambiente clínico, para acadêmicos, funcionários e docentes do curso, de forma a servir como roteiro ou protocolo para o atendimento clínico. Desse modo, visaria à proteção tanto dos discentes, docentes e funcionários quanto dos usuários das clínicas odontológicas da Unimontes, já que existem possibilidades de infecção (cruzada ou não).

Entretanto, com a situação de pandemia pela COVID-19, que impactou a maneira de viver de boa parte da população mundial nos últimos meses, a Comissão se viu com a necessidade de alterar a forma de atendimento clínico, já estruturada e conhecida, para pensar numa nova rotina de trabalho, tendo em vista o risco de infecção pelo novo coronavírus.

Desse modo, embora existisse um manual previamente estabelecido e normatizado para o Curso de Odontologia, tornou-se necessário o desenvolvimento de um novo documento, com base nas evidências científicas que já estavam disponíveis. Então, a Comissão de Biossegurança e Infraestrutura esforçou-se em realizar uma análise criteriosa 
da literatura, leis, portarias e normas técnicas do Ministério da Saúde, Secretarias Estaduais e Municipais, Protocolos de Biossegurança de outras Instituições de Ensino Superior - IES e de Entidades de Classe. E, após diversas reuniões online, remotamente, no período de março a julho de 2020, foi elaborado o Protocolo de Biossegurança do Curso de Odontologia da Unimontes em Tempos de COVID-19, com o intuito de normatizar e organizar o processo de trabalho, assim como estabelecer protocolos seguros ao atendimento odontológico nas clínicas e às práticas laboratoriais, nesse contexto atual de pandemia.

O protocolo foi baseado no Consenso ABENO: Biossegurança no ensino odontológico pós-pandemia da COVID-19, proposto pela Associação Brasileira de Ensino Odontológico - ABENO, o qual teve a colaboração de IES de todo o país, na área da Odontologia $^{8}$. Uma vez feito o diagnóstico da situação das clínicas e laboratórios do Centro de Ciências Biológicas e da Saúde - CCBS da Unimontes, realizaram-se adaptações e adequações no que foi apresentado pelo Consenso ABENO e outras fontes, fundamentais à realidade encontrada no CCBS.

Esse instrumento conta, em suma, com orientações e recomendações acerca de como se deve organizar o ambiente clínico, com estratégias de controle de aerossóis, lista de insumos a ser adquiridos e utilizados, entre outros; do fluxo de usuários e acompanhantes, durante as consultas; de como deve ser feita a sala de espera e como proceder se o usuário apresentar ou relatar sintomas de COVID-19; da limpeza, acondicionamento e esterilização dos instrumentais odontológicos, limpeza do ambiente clínico e gerenciamento dos resíduos de saúde; da maneira pela qual os discentes, docentes e funcionários devem se portar ao sair e retornar para casa; da descrição de paramentação e desparamentação correta e específica para acadêmicos, professores, técnicos em Saúde Bucal e funcionários de serviços gerais, durante a utilização do espaço.

O documento redigido obteve reconhecimento pela equipe de protocolo de retorno da Universidade, em função da consolidação clara e precisa das informações encontradas, a fim de se fazer o cuidado à saúde com a segurança necessária, ao retomarem as atividades presenciais nos tempos de COVID-19. 


\section{DISCUSSÃO}

Biossegurança diz respeito a um grupamento de intervenções que se destinam a controlar, precaver, amenizar ou ainda eliminar possíveis riscos à qualidade de vida, saúde humana e do meio ambiente, constituindo uma estratégia imprescindível para o desenvolvimento, promoção e manutenção do bem-estar e proteção à vida, especialmente na área da saúde $e^{4,9}$.

Dentre as determinações contidas no Código de Ética Odontológica, no artigo $5^{\circ}$, incisos V e VII, zelar pela saúde dos pacientes, bem como promover a saúde coletiva no desempenho de suas funções, constituem deveres fundamentais dos cirurgiões-dentistas e outros profissionais de Odontologia ${ }^{10}$.

As clínicas de ensino de Odontologia constituem um ambiente de alto risco, no que se diz respeito à infecção cruzada, uma vez que, com elevada frequência, reúnem em um só momento dezenas de pessoas em um mesmo local. Isso requer que medidas sérias e efetivas sejam adotadas, com o intuito de reduzir o risco de infecções, tornando o ambiente clínico mais seguro tanto para profissionais, quanto para usuários ${ }^{11-15}$ Com o propósito de reunir informações para a implementação dessas medidas, no âmbito da Odontologia, várias entidades da Odontologia mundial se alinharam e entraram em consenso ${ }^{16}$ (Quadro 1).

Quadro 1 - Consenso entre diversas entidades odontológicas para adequação da clínica odontológica durante a pandemia da COVID-19.

Minimização de pacientes na sala de espera.

Disposição das cadeiras com uma distância mínima de 1 metro.

Limitação dos pacientes que acompanham pacientes na sala de espera.

Uso de máscaras adequada dos pacientes, acompanhantes e equipe.

Fornecimento álcool 70\% gel aos pacientes em todos os ambientes da clínica.

Remoção dos objetos da sala de espera que possam permitir infecção cruzada.

Instalação da barreira de acrílico entre área de recepção e paciente.

Ventilação do ambiente constante, preferencialmente com janelas abertas.

Realizar atendimento odontológico em salas individuais sempre que possível.

Instalação de divisórias em cadeiras em ambientes de plano aberto.

Distância mínima de 2 metros entre cada cadeira de plano aberto.

Triagem remota prévia ao agendamento. 
Verificação de temperatura dos pacientes, previamente, ao atendimento.

Enxaguatório bucal antisséptico pré-procedimento.

Exposição mínima de objetos, instrumentos e materiais na sala do paciente, durante atendimento odontológico.

Uso de barreiras físicas que são constantemente tocadas com frequência e equipamentos de difícil limpeza.

Uso de equipamentos de proteção individual por todos os que estejam na sala clínica.

Evitar ou minimizar o uso de dispositivos geradores de aerossol.

Priorizar a utilização de instrumentos manuais (evitar equipamentos ultrassônicos).

Evitar a tomada de radiografias intrabucais.

Utilizar isolamento absoluto com dique de borracha sempre que possível.

Utilização de sucção de bomba a vácuo para aspiração interna da boca - mitigação do aerossol.

Trabalho a quatro mãos.

Descarte adequado de resíduos infecciosos após cada atendimento.

Esterilização de todos os instrumentos críticos, incluindo peças de mão.

Ventilação da sala clínica para dispersão/eliminação de aerossol a cada atendimento (tempo médio de 40 minutos).

Limpeza e desinfeção da sala clínica após cada atendimento.

Uso de roupa de trabalho exclusiva, lavada separadamente.

Durante a fase de retomada do atendimento odontológico eletivo, muitas medidas devem permanecer, dentre todas as orientações a respeito da segurança no atendimento odontológico, existe um consenso especial sobre:

A necessidade de realizar triagem dos pacientes antes, durante agendamento (remoto) e atendimento odontológico em que os horários devem permitir intervalos mais longos entre os pacientes para permitir a purga dos aerossóis, limpeza da sala e evitar aglomeração.

Os requisitos indicados para instalação da clínica odontológica:

Medidas de mitigação de aerossol e renovação/filtração do ar;

Uso rigoroso de equipamentos de proteção individual (EPIs) no atendimento clínico;

Considerar todos os pacientes como assintomáticos da COVID- 19.

Fonte: Adaptado de Borges $(2020)^{16}$.

Devido a suas particularidades, o ambiente odontológico possibilita que o ar seja uma via potencial de transmissão de microrganismos, por meio das gotículas e dos aerossóis, que podem contaminar diretamente o profissional ao atingirem a pele e a mucosa, por inalação e ingestão, ou indiretamente, quando contaminam as superfícies. As gotículas e os aerossóis são gerados durante a tosse, espirro e fala ou são provenientes dos instrumentos rotatórios, seringas tríplices, equipamentos ultrassônicos e por jateamento. As gotículas são consideradas de tamanho grande e podem atingir até um metro de distância. Por serem 
pesadas, rapidamente se depositam nas superfícies. Os aerossóis são partículas pequenas lançadas, com menos de 50 mícrones de diâmetro, que podem permanecer suspensas no ar durante horas e serem dispersas a longas distâncias, atingindo outros ambientes, carreadas por correntes de $\operatorname{ar}^{6,17-19}$.

Diante dessas considerações e conhecendo-se as formas de contágio do novo coronavírus, percebe-se sua alta capacidade de transmissão no meio odontológico ${ }^{5,6}$. Neste sentido, uma das questões centrais do protocolo desenvolvido pela Comissão de Biossegurança e Infraestrutura do Curso de Odontologia da Unimontes foi quanto ao controle dos aerossóis gerados durante os atendimentos.

Entre os pontos abordados para esse controle, incluíram-se a limitação do atendimento a um único usuário por turno, por acadêmico, tendo em vista que o fluxo de usuários rotineiramente era alto e por períodos prolongados; a utilização de sucção/aspiração contínua de alta potência, além de isolamento absoluto sempre que possível; evitar o uso de canetas de alta e baixa rotação e aparelhos que gerem aerossóis, como jato de bicarbonato e ultrassom, porém se necessário, dar preferência a canetas com válvula antirrefluxo e regular o volume de saída de água; evitar o uso de seringa tríplice, especialmente na combinação ar/água (spray), utilizando, como alternativa, seringas descartáveis com soro e, para secar, alta sucção e/ou compressas de gaze; priorizar o trabalho a quatro mãos, mas com possibilidade de trabalho a seis mãos, com a terceira pessoa circulante, similar a centros cirúrgicos $^{12}$.

Além disso, a recomendação fundamental de evitar o contato direto do profissional, por aspiração, com os aerossóis gerados é o uso de respirador N95, PFF2 ou similares sem válvula, os quais foram abordados nos itens destinados à paramentação e sua colocação correta foi ilustrada, com orientação sobre o teste de vedação da mesma, para que fosse o mais didático possível aos leitores do protocolo. Essas máscaras de proteção respiratórias têm um grande potencial de filtração, sendo que a N95, por exemplo, filtra 95\% de partículas aéreas, o que faz com que sejam bastante úteis em ambientes com alta produção de spray ou respingos contaminados com saliva ou sangue ${ }^{5,19}$. Entretanto, a proteção do profissional não se restringe apenas ao uso dos respiradores. O mesmo deve estar paramentado devidamente, com todos os equipamentos de proteção individuais (EPIs) recomendados, como gorro, óculos de proteção, protetor facial (face shield), pijama 
cirúrgico, avental impermeável descartável, luvas de procedimento, calçado cirúrgico específico 5 .

A limpeza e descontaminação de superfícies são atitudes básicas e fundamentais para o controle da disseminação de infecções. Nos serviços de saúde, visam garantir aos usuários um ambiente com a menor carga de contaminação possível, diminuindo a transmissão de infecções ${ }^{20}$. Foram levados em conta, no protocolo, determinados produtos e maneiras de aplicação, conforme a necessidade e a indicação (Quadro 2).

Quadro 2 - Comparativo de desinfetantes de superfícies.

\begin{tabular}{|c|c|c|c|c|}
\hline Desinfetante & Álcool a 70\% & $\begin{array}{l}\text { Hipoclorito de } \\
\text { Sódio a } 1 \%\end{array}$ & $\begin{array}{l}\text { Ácido Peracético } \\
(0,2 \text { a } 0,5 \%)\end{array}$ & $\begin{array}{c}\text { Quaternários de } \\
\text { Amônio 5 } 5^{\mathrm{a}} \text { Geração } \\
\text { com Biguanida (7 a } \\
9 \% \text { 1:200) }\end{array}$ \\
\hline Nível & Médio. & Médio. & Alto. & Alto. \\
\hline Aplicação & $\begin{array}{c}\text { Fricção em } 3 \text { etapas } \\
\text { intercaladas pelo } \\
\text { tempo de secagem } \\
\text { natural, totalizando } \\
10 \text { segundos. }\end{array}$ & $\begin{array}{c}\text { Aplicação na } \\
\text { superfície por } 2 \text { a } 5 \\
\text { segundos. }\end{array}$ & $\begin{array}{l}\text { Aplicação na } \\
\text { superfície por tempo } \\
\text { indicado pelo } \\
\text { fabricante. }\end{array}$ & $\begin{array}{l}\text { Aplicação na superfície, } \\
\text { deixar agir por } 10 \\
\text { segundos e remover com } \\
\text { papel descartável. }\end{array}$ \\
\hline Vantagens & $\begin{array}{l}\text { Fácil aplicação, ação } \\
\text { rápida, compatível } \\
\text { com artigos } \\
\text { metálicos, superfícies } \\
\text { e tubetes anestésicos. }\end{array}$ & $\begin{array}{c}\text { Ação rápida, indicado } \\
\text { para superfícies e } \\
\text { artigos não metálicos } \\
\text { e materiais } \\
\text { termossensíveis. }\end{array}$ & $\begin{array}{l}\text { Não forma resíduos } \\
\text { tóxicos, efetivo na } \\
\text { presença de matéria } \\
\text { orgânica, rápida ação } \\
\text { em baixa temperatura, } \\
\text { indicado para } \\
\text { superfícies e artigos } \\
\text { não metálicos. } \\
\end{array}$ & $\begin{array}{l}\text { Fácil aplicação, compatível } \\
\text { com artigos metálicos, } \\
\text { estável, baixa toxicidade. }\end{array}$ \\
\hline Desvantagens & $\begin{array}{l}\text { Volátil, inflamável, } \\
\text { resseca plásticos e } \\
\text { opacifica acrílico. }\end{array}$ & $\begin{array}{l}\text { Instável, corrosivo, } \\
\text { inativado por } \\
\text { matérias orgânicas, } \\
\text { irritação de pele e } \\
\text { mucosas. }\end{array}$ & $\begin{array}{l}\text { Instável quando } \\
\text { diluído, corrosivo } \\
\text { para alguns tipos de } \\
\text { metais, odor. }\end{array}$ & Custo. \\
\hline
\end{tabular}

Fonte: Adaptado de ABENO (2020) ${ }^{8}$.

Uma limitação vivenciada no presente estudo foi a incompatibilidade de todos os membros da Comissão estarem presentes, simultânea e remotamente, em todas as reuniões realizadas, embora todos tenham contribuído tanto com a elaboração do protocolo, quanto com a atuação da Comissão durante o momento de pandemia. Além disso, diante da velocidade de descobertas e produções científicas sobre a COVID-19, atualizações constantes foram necessárias ao protocolo e continuarão a ser. 


\section{CONCLUSÃO}

A Comissão de Biossegurança e Infraestrutura das Clínicas e Laboratórios do Curso de Odontologia da Unimontes cumpre com uma missão não só de atender às exigências legais, mas de orientar todo o corpo docente e discente, bem como os funcionários, no sentido de se realizar as atividades no ambiente clínico odontológico com segurança e proteção, considerando a responsabilidade social e a formação profissional. Os cuidados à saúde, muitas vezes negligenciados pelo cirurgião-dentista, são de extrema importância para reduzir o risco de infecção cruzada, sobretudo em tempos de COVID-19. Desse modo, a atuação da Comissão e a criação de um protocolo de biossegurança específicos para a realidade de pandemia buscam a efetividade das ações a serem empregadas, com capacitações, materiais educativos e treinamentos, a fim de se propagar o conhecimento e a sua adequada aplicação para o retorno das atividades presenciais.

\section{AGRADECIMENTOS}

À Camila Lages Bento, pelo apoio técnico.

\section{REFERÊNCIAS}

1 BEZERRA, André L. D. et al. Biossegurança na odontologia. ABCS Health Sciences, v. 39, n. 1, p. 29-33, 2014.

2 NOGUEIRA, Sumaia A.; BASTOS, Luciana F.; COSTA, Iris C. C. Riscos ocupacionais em odontologia: revisão da literatura. UNOPAR Científica, v. 12, n. 3, p. $11-20,2010$.

3 BRASIL. Ministério da Saúde. Agência Nacional de Vigilância Sanitária. Serviços odontológicos: prevenção e controle de riscos. Brasília: Ministério da Saúde, 2006. 156 p. (Série A. normas e manuais técnicos).

4 ALVES, Leandro S.; PACHECHO, Jonas S. Biossegurança - fator determinante nas unidades de atendimento à saúde. Revista Fluminense de Extensão Universitária, v. 5, n. 1, p. 33-40, 2015. 
5 FRANCO, Juliana B.; CAMARGO, Alessandra R.; PERES, Maria P. S. M. Cuidados odontológicos na era do COVID- 19: recomendações para procedimentos odontológicos e profissionais. Revista da Associação Paulista de Cirurgiões Dentistas, v. 74, n. 1, p. 18-21, 2020.

6 CABRERA-TASAYCO, Fiorella P. et al. Biosafety measures at the dental office after the appearance of COVID-19: a systematic review. Disaster Medicine and Public Health Preparedness, p. 1-5, 2020.

7 UNIVERSIDADE ESTADUAL DE MONTES CLAROS (UNIMONTES). Gabinete do Reitor. Portaria no 094 - Reitor/2010. Constitui a Comissão de Biossegurança e Infra-estrutura das Clínicas e Laboratórios do Curso de Odontologia, vinculado ao Centro de Ciências Biológicas e da Saúde (CCBS). Montes Claros: Unimontes, 2010.

8 ASSOCIAÇÃO BRASILEIRA DE ENSINO ODONTOLÓGICO (ABENO). Consenso Abeno: biossegurança no ensino odontológico pós-pandemia da COVID-19. Porto Alegre: ABENO, 2020. 86 p.

9 BRASIL. Ministério da Saúde. Biossegurança em saúde: prioridades e estratégias de ação. Brasília: Ministério da Saúde, 2010.

10 CONSELHO FEDERAL DE ODONTOLOGIA (CFO). Código de Ética Odontológica - aprovado pela Resolução CFO-118/2012. Brasília: CFO, 2012.

11 WORLD HEALTH ORGANIZATION (WHO). Getting your workplace ready for COVID-19. Geneva: WHO, 2020a. Disponível em: https://www.who.int/docs/defaultsource/coronaviruse/getting-workplace-ready-for-covid-19.pdf?ua=1. Acesso em: 14 mar. 2020.

12 BRASIL. Agência Nacional de Vigilância Sanitária. Nota técnica $n^{o}$. 04, de 05 de maio de 2020. Orientações para serviços de saúde: medidas de prevenção e controle que devem ser adotadas durante a assistência aos casos suspeitos ou confirmados de infecção pelo novo coronavírus (SARS-CoV-2). Brasília: Diário Oficial da União, 2020. Disponível em:

https://www20.anvisa.gov.br/segurancadopaciente/index.php/alertas/item/nota-tecnican-04-2020-gvims-ggtes-anvisa-atualizada-em-17-02-2020. Acesso em: 18 maio 2020.

13 AMERICAN DENTAL ASSOCIATION (ADA). Return to work interim guidance toolkit. Chicago: ADA, 2020.

14 CONSELHO FEDERAL DE ODONTOLOGIA (CFO). COVID19: manual de boas práticas em biossegurança para ambientes odontológicos. Brasília: CFO, 2020. Disponível em: http://website.cfo.org.br/covid19-\%20manual-de-boas-praticas-embiosseguranca-para-ambientes-odontologicos-e-lancado-com-apoio-institucional-docfo/. Acesso em: 05 maio 2020. 
15 BARBIERI, Ana A. et al. Biosafety measures in dental practice: literature review. Brazilian Dental Science, v. 22, n. 1, p. 9-16, 2019.

16 BORGES, Lusiane. O novo normal da odontologia. Jornal da Associação Brasileira de Odontologia, São Paulo, 31 ago. 2020. Caderno 1, p. 20-22.

17 PENG, Xian et al. Transmission routes of 2019-nCoV and controls in dental practice. International Journal of Oral Science, v. 12, n. 9, p. 1-6, 2020.

18 DOREMALEN, Neeltje et al. Aerosol and surface stability of SARS-CoV-2 as compared with SARS-CoV-1. The New England Journal of Medicine, v. 382, n. 16, p. 1564-1567, 2020.

19 SILES-GARCIA, Adriana A. et al. Biosafety for dental patients during dentistry care after COVID-19: a review of the literature. Disaster Medicine and Public Health Preparedness, p. 1-6, 2020.

20 WORLD HEALTH ORGANIZATION (WHO). Cleaning and disinfection of environmental surfaces in the context of COVID-19. Geneva: WHO, 2020b. Disponível em: https://www.who.int/publications/i/item/cleaning-\%20and-disinfectionof-environmental-surfaces-inthe-context-of-covid-19. Acesso em: 14 mar. 2020. 